\title{
Clinicopathological, Endoscopic and Radiological Evaluation of Intestinal Tuberculosis among Nepalese Patients at a Tertiary Care Center
}

\author{
Tshering Wangdi Sherpa ${ }^{1}$, Rahul Pathak ${ }^{2}$, Anurag Jha ${ }^{3}$
}

\section{Author Info:}

${ }^{1}$ Department of Medicine, Shree Birendra Hospital, Nepal, tshering.ongdi@gmail.com

${ }^{2}$ Department of Gastroenterology, TUTH, Nepal, pathak.drrahul@ googlemail.com

${ }^{3}$ Department of

Gastroenterology, TUTH, Nepal, drjhanurag@gmail.com

\section{*Corresponding Author:}

Dr. Tshering Wangdi Sherpa, Consultant gastroenterologist

Email/Contact:

tshering.ongdi@gmail.com

00977-9851146166

\section{ABSTRACT}

Background: Intestinal tuberculosis is often difficult to diagnose due to its insidious onset and nonspecific and protean manifestations. Delay or failure of diagnosis is associated with significant complications like perforation, abscess, fistula and strictures. The objective of the study was to provide a descriptive analysis of clinical, endoscopic, radiological and pathological findings in these patients.

Methods: A prospective study including 52 hospitalized patients was done between April 2018 to 2020 at Tribhuwan University Teaching Hospital. Statistical analyses were done by Microsoft excel version 16.

Results: Males were $63 \%$ while females were $37 \%$. The average age was 37 years. $84 \%$ had abdominal pain followed by abdominal distension (50\%), chronic diarrhea $(51 \%)$ and cough $(21 \%)$. Abnormal chest X-Ray was found in (37\%) among whom $(53 \%)$ had sputum positive for tubercular Bacilli. Common CT scan findings were thickening and heterogenous enhancement of bowel loops (79\%), lymphadenopathy (56\%) and ascites (44\%). Endoscopically, commonly involved sites were terminal Ileum (95\%), cecum (77\%) and ascending colon (42\%). Frequent finding were ulcers (83\%), patulous and deformed Ileocecal valve (33\%), mucosal nodularity (38\%), hypertrophic mucosa $(21 \%)$ and strictures (11\%). Biopsies revealed granulomas in $(77 \%)$ specimens. (65\%) of them had central caseous necrosis. Among 10 subjects undergoing needle aspiration of lymphnodes, (70\%) had central caseous necrosis. Those with coexisting peritoneal involvement had a mean ascitic fluid adenosine deaminase level of $73 \pm 8 \mathrm{IU} / \mathrm{L}$

Conclusion: Intestinal tuberculosis remains a major concern in developing countries. A composite analysis of the clinical manifestations, CT scan, endoscopic findings and pathological examination of the biopsies aid in making a proper and timely diagnosis so as to prevent complications and mortality.

Keywords: CT scan, Diagnosis,endoscopy,Intestinal tuberculosis

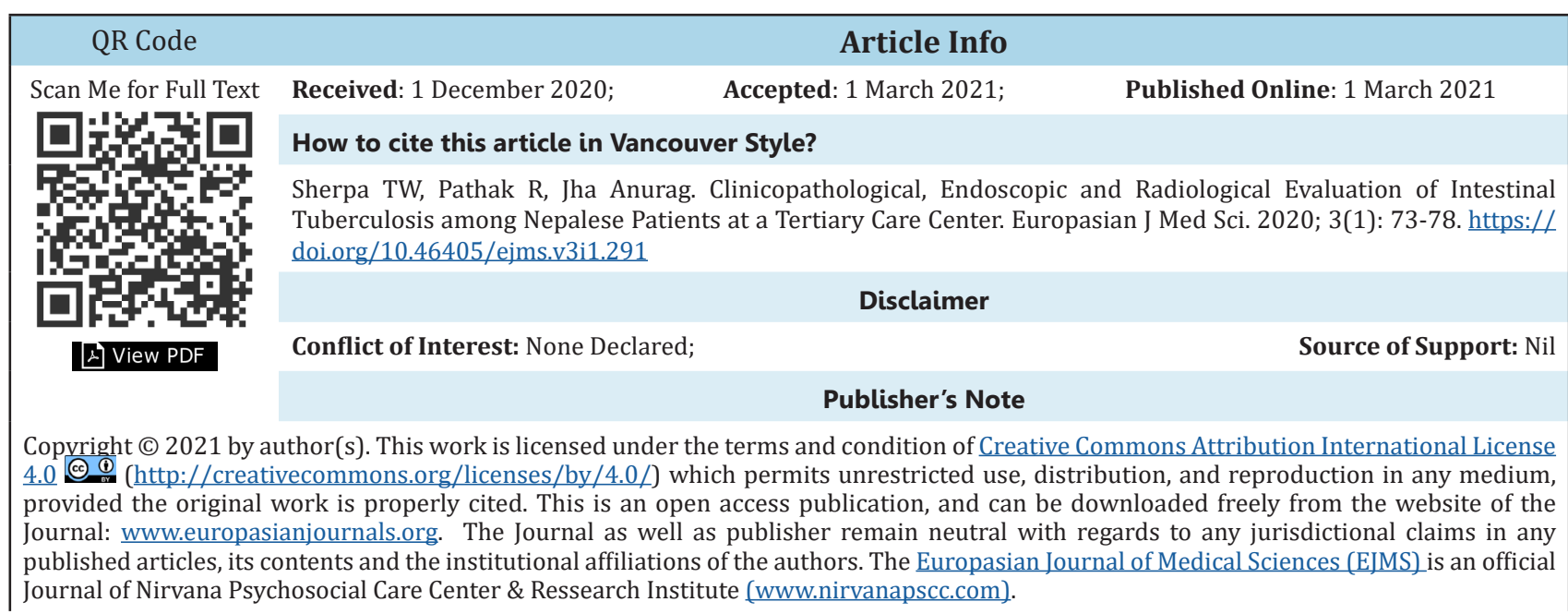




\section{INTRODUCTION}

Tuberculosis (TB) is one of the leading infectious cause of death worldwide, more so in the developing countries. According to the World Health Organization (WHO), in 2018, 10.0 million individuals became infected with TB with a mortality of 1.7 million ${ }^{1}$. Extrapulmonary tuberculosis accounted for $15 \%$ of all cases. Though the true prevalence of intestinal tuberculosis (ITB) is not known, it is highly common in developing countries like Nepal.

Patients with ITB present with protean manifestations of variable duration with abdominal pain being the most common symptom. Due to the nonspecific nature of presentation and laboratory investigations, diagnosis can be delayed and often misdiagnosed leading to unwanted morbidity and mortality $^{2}$. A definite diagnosis of ITB can be made if any of the following criteria are met: histology showing tubercles with caseating necrosis, consistent histology from mesenteric lymph nodes, culture showing growth of Mycobacterium tuberculosis (MTB) or histology showing acid-fast bacilli (AFB) in the lesion ${ }^{3}$. However, all these above tests have low sensitivity and diagnosis cannot be readily excluded if they are negative.

TB can virtually affect any part of the gastrointestinal tract, with the ileocecal region being the most common. This is due to its narrow lumen, relative stasis allowing digestion of the mycobacteria's fatty capsule and efficient absorption of the organism and abundant lymphatics in the ileocecal region ${ }^{4,5}$. Ileocecal TB often mimics Crohn's disease and differentiating between the two is imperative as the use of immunosuppressants like glucocorticoids and infliximab which are the cornerstone of therapy in Crohn's disease may have a detrimental effect in patients with tuberculosis ${ }^{6}$. The second most common location in the GIT is the colon, while the stomach and esophagus are rarely involved.

Due to the non-specific symptoms and protean manifestations, diagnosis of ITB requires a high index of suspicion. A comprehensive radiological and endoscopic evaluation should be done in these patients and whenever possible, biopsy specimens should be obtained for histopathological examination so that a proper diagnosis can be made timely. This study aims to evaluate the clinical, radiological, endoscopic and pathological features of ITB among Nepalese patients.

\section{MATERIALS AND METHODS}

This was a prospective, observational study conducted between February 2018 to 2020 at a tertiary care center. The study was approved by Institutional review Committee of Institute of Medicine (IOM), Tribhuwan University. All patients aged 16 and above who had clinical, radiological, endoscopic and pathological features of ITB were included in the study. Written informed consents were obtained from all patients for endoscopy and participation in the study. Non-probability sampling method was used and all consecutive patients meeting the inclusion criteria were included during the study period of 2 year. A total of 52 patients were included.

Patients who had suggestive clinical features underwent relevant investigations as per routine protocol. Findings of chest X- Ray and Contrast enhanced CT (CECT) of abdomen were recorded to include in the radiological evaluation. Endoscopy was done in all patients and biopsies were taken from intestinal lesions for histopathology. Computed Tomography (CT) guided Fine Needle Aspiration (FNA) of regional lymphnodes were done where necessary. In those which coexisting peritoneal tuberculosis, USG guided aspiration of ascitic fluid was done for biochemical analysis. A definite diagnosis of ITB was based on the following criteria's: 1) histology showing epithelioid granulomas with caseation 2) AFB seen on Zeil-Neilson's stain 3) suggestive extraintestinal involvement including sputum positive pulmonary tuberculosis and peritoneal involvement with high Adenosine Deaminase (ADA) values.

Data collection was done using de novo written proformas. All data were collected by the principle investigator. Statically analysis was done using Microsoft excel version 16. Descriptive data with normal distribution were expressed as mean \pm standard deviation.This i

\section{RESULTS}

\section{Clinical Features}

The mean age of the patients was $37 \pm 15$ years. $33(63 \%)$ patients were males while 19 (37\%) were females with a male to female ratio of 1.7:1. Abdominal pain was the most common symptom that was present among $44(84.61 \%)$ patients. 26 (50\%) of them complained of abdominal distension. Constitutional symptoms like fever and loss of weight with loss of appetite were found in 42 (81\%) and $44(84.61 \%)$ patients respectively. Cough due to pulmonary or pleural involvement was present in $22(42.30 \%)$ subjects. Due to bowel involvement, 27 (51.91\%) of them had chronic diarrhea among whom 8 (15.38\%) had blood mixed diarrhea while 5 $(9.61 \%)$ patients had altered bowel habits. 
Table 1: symptom distribution

\begin{tabular}{lll}
\multicolumn{1}{c}{ Symptoms } & N & Percent \\
\hline Abdominal Distension & 26 & $50 \%$ \\
Abdominal Pain & 44 & $84.61 \%$ \\
Fever & 42 & $81 \%$ \\
Cough & 22 & $42.30 \%$ \\
Chronic diarrhea & 27 & $51.91 \%$ \\
Blood mixed diarrhea & 8 & $15.38 \%$ \\
Loss of appetite and & 44 & $84.61 \%$ \\
weight & & $9.61 \%$ \\
Altered bowel habit & 5 & \\
\hline
\end{tabular}

\section{Radiological Findings}

Most patients, 28(54\%) had a normal chest X-Ray. In those with an abnormality, Active parenchymal lesions were the most common finding present in $19(36.53 \%)$ patients, among whom $10(52.63 \%)$ had sputum smear positive for AFB. Similarly, 4 (7.69\%) of them had pleural effusions while old fibrotic changes were noted in 2 (3.84\%).

$40(79 \%)$ patients had thickening and heterogenous enhancement of the bowel loops in CT Scan of the abdomen. Other patterns of intestinal involvement included pulled up cecum in $5(9.61 \%)$ and clumping of bowel loops in $8(15.38 \%)$ patients. The most common site of involvement was the terminal Ileum, followed by IC valve, caecum, ascending and Table 2: CT scan findings

\begin{tabular}{lll}
\hline CT Findings & $\mathrm{N}$ & percentage \\
\hline $\begin{array}{l}\text { Thickening and } \\
\text { enhancement of } \\
\text { bowel wall }\end{array}$ & 41 & $78.84 \%$ \\
$\begin{array}{l}\text { Pulled up caecum } \\
\text { Ascites }\end{array}$ & 5 & $9.61 \%$ \\
$\begin{array}{l}\text { Lymphadenopathy } \\
\text { Clumping of bowel }\end{array}$ & 29 & $44 \%$ \\
$\begin{array}{l}\text { loops } \\
\text { Spleenomegaly w/ } \\
\text { infiltrates }\end{array}$ & 1 & $15.76 \%$ \\
\end{tabular}

Table 3: Endoscopic Findings

\begin{tabular}{lll}
\hline Findings & Frequency & Percentage \\
\hline $\begin{array}{l}\text { Ulcers } \\
\begin{array}{l}\text { Patulous and } \\
\text { deformed IC } \\
\text { valve }\end{array}\end{array}$ & 43 & $82.69 \%$ \\
$\begin{array}{l}\text { Mucosal } \\
\text { nodularity }\end{array}$ & 20 & $32.69 \%$ \\
$\begin{array}{l}\text { Mucosal } \\
\text { erosions }\end{array}$ & 6 & $38.46 \%$ \\
$\begin{array}{l}\text { Hypertrophied } \\
\text { mucosa }\end{array}$ & 11 & $11.53 \%$ \\
Strictures & 6 & $21.15 \%$ \\
\hline
\end{tabular}

transverse colon in decreasing frequency. Pancolonic involvement in CT was noted in one patient. Extraintestinal involvement was seen in almost all patients with intraabdominal lymphadenopathy in $29(55.76 \%)$, ascites in $23(44 \%)$ and mesentric and omental thickening in $8(15.38 \%)$ patients. Mesenteric lymphnodes were involved in the majority followed by retroperitoneal, paraaortic, aortocaval and portal group. Lymph nodes forming conglomerate masses were found among $9(17 \%)$ subjects while 25 (47\%) of them had central necrotic areas on $\mathrm{CT}$.

\section{Endoscopy}

Gastroscopy was done in one patient with duodenal involvement while the remaining underwent colonoscopy. 43 (82.69\%) patients had ulcers on endoscopy. Majority of the patients had multiple ulcers which were predominantly transversely oriented, discrete and variable in size and depth with well circumscribed margins. Other common findings were patulous and deformed IC valve in 17 (32.69\%) and mucosal nodularity in $20(38.46 \%)$ subjects. Edematous and hypertrophic mucosa leading to formation of mass like lesion were noted among 11 (21.15\%) patients while strictures were noted in 6 (11.53\%). In regards to the site of the lesions, the terminal ileum was involved in 41 (95.34\%) patients followed by the caecum in 33 (76.74\%), Ascending colon in 18 (41.85\%), transverse colon in 7 (16.27\%) 
and descending colon in 5 (11.62\%) patients. 2 $(4.65 \%)$ patients with disseminated tuberculosis had pancolonic involvement including the rectum while the duodenum was involved in $1(2.32 \%)$ patient. Multiple biopsies for histopathological examination were taken from the ulcer margin and base. In those without ulcers, biopsies were taken from erosions and nodular mucosa.

\section{Histopathological and biochemical findings}

$40(76.92 \%)$ patients had granulomas in the biopsy specimens from intestinal lesions. Among those specimens with granulomas, 26 (65\%) had central caseous necrosis. Majority of the granulomas were well-formed with 21 (52.5\%) of them being multiple. AFB was detected in $9(22.5 \%)$ specimens. Other findings included chronic inflammatory ileitis or colitis in $12(23.07 \%)$ and cryptitis and crypt abscesses in 3 (5.76\%) specimens. 10 (19.23\%) patients, in whom a definite diagnosis could not be established underwent CT guided FNAC of mesenteric lymph nodes. 7 (70\%) of them had granulomas with central caseous necrosis and AFB was positive in 3 (30\%) specimens. 23 (44.23\%) patients had coexisting peritoneal tuberculosis. Ascitic Fluid analysis was done in all of these patients. All subjects had low SAAG, high protein ascites with an average WBC count of $2120 \pm 430$ and predominant lymphocytosis of $74 \pm 3 \%$. The mean ADA value in all patients were $73 \pm 8 \mathrm{IU} / \mathrm{L}$ with a minimum value of $46 \mathrm{IU} / \mathrm{L}$.

\section{DISCUSSION}

Intestinal TB can affect any individual irrespective of age or gender. Globally, among HIV negative patients with tuberculosis, $57 \%$ were males and $32 \%$ were females with the remaining $11 \%$ being children ${ }^{1}$. Our study had a similar finding with $63 \%$ of patients being male and the remaining $37 \%$ being females. Majority of our patients were of the reproductive age group between 20 to 49 years. This was similar to the data of the Nepal tuberculosis program, where TB was most prevalent between 15 to 44 years of age accounting for $47 \%$ of all cases ${ }^{7}$.

The clinical manifestations of ITB are protean and nonspecific. Hence, they are often misdiagnosed as other intestinal illnesses like crohn's disease, lymphoma etc. In a retrospective study of 250 patients by Patel et al, abdominal pain occurred in $79 \%$ of the patients. This was followed by the constitutional symptoms of fever and weight loss. Chronic diarrhea occurred in $22 \%$ of the patients ${ }^{8}$. In another similar series from China, abdominal pain found in $87 \%$ of patients as the commonest symptom followed by fever and weight loss. Chronic diarrhea accounted for $47 \%$ of the symptoms ${ }^{9}$. These characteristics commiserate with the findings of this study.

Chest X-Ray is routinely done in all patients with suspected tuberculosis. In a meta-analysis including 1002 patients, an abnormal chest X- Ray was found in an average of $38 \%$ of studies ${ }^{10}$. Marshall et al, in their review found that concomitant active pulmonary TB is found only among $15-20 \%$ of the patients with abdominal tuberculosis ${ }^{11}$. The findings in our study were not very dissimilar these. In general, CT is the most useful imaging to evaluate intestinal tuberculosis as it provides a comprehensive detail of intraabdominal structures and lesions and helps the clinician identify the site of the lesion. Though barium studies were widely used in the past, the have been largely replaced by CT scan as it has the ability to identify both intraluminal and extramural abnormalities. CT imaging in the setting of ITB demonstrate bowel wall thickening and mucosal enhancement, ascites, lymph nodes, thickening of the mesentery, omentum and the peritoneum, strictures and occasionally intraluminal lesions $s^{5,12,13}$. In regards to site, the ileocecal region is most commonly involved. The lymph nodes most commonly involved include mesenteric, peri-portal, peri-pancreatic, and upper para-aortic groups of nodes $^{12,14}$. Retroperitoneal lymphnodes are rarely involved. However, in our study the mesenteric, retroperitoneal, paraaortic and aortocaval nodes in decreasing order were most commonly involved. Presence of conglomerate masses of lymphnodes and the presence of central necrotic areas are more favorable towards $\mathrm{TB}^{15}$, and these findings were also demonstrated in our study.

The fact that direct visualization of the intestinal lumen is possible and necessary biopsies can be taken makes endoscopy a must in the diagnosis of ITB. The characteristic lesions include ulcers which are linear, transverse or circumferential with overlaying exudates. The surrounding mucosa is usually abnormal with erythemia, oedema and nodularity ${ }^{16}$. In a study from Korea including 42 patients, the most common endoscopic findings were hyperemic mucosa, ulcers usually transverse or annular and hypertrophic mucosa which were found in 42 (100\%), 39 (93\%) and 13 (31\%) patients respectively ${ }^{17}$. In our study, ulcers which were mostly transverse or circumferential and discrete were the most common findings followed by patulous and deformed IC valve, mucosal nodularity, hypertrophic mucosa and strictures. These findings were 
comparable to a large study including 250 patients from India ${ }^{8}$. However, endoscopic features of ITB are also not pathognomic. In fact, lesions of ITB can often be confused with Crohn's disease. Crohn's disease is characterized by longitudinal ulcers, aphthous ulcers, cobblestone appearance and mucosa surrounding the ulcers is healthy ${ }^{18}$. Differentiation between the two is of particular importance specially in south Asian countries like Nepal and India where the incidences of Crohn's disease is increasing but tuberculosis remains prevalent.

Ultimately, the diagnosis will be based on the characteristic histopathology findings in biopsy specimens. Tubercular granulomas are usually large (> 200 microns), confluent, dense (> 5-10/hpf), located in submucosa, and are characterized by central caseation, which is diagnostic and exclusive for tuberculosis ${ }^{19}$. The yield from biopsies is not always good due to the paucicellular nature of ITB and the predominance of granuloma in the deeper layers. Hence multiple, deep biopsies should be taken from the lesions to increase the diagnostic yield. In a study by $\mathrm{N}$ patel et al, caseating granulomas and AFB smear positive were found in $66 \%$ and $29 \%$ of cases, respectively ${ }^{8}$. Harvarth et al., in their study demonstrated the sensitivity of positive histology as $41 \%$ and positive histology with negative smear of $70 \%$ in diagnosing ITB $^{20}$. In our study, positive histology in the form of central caseous necrosis was found in $65 \%$ of patients with granulomas while $22.5 \%$ of the patients had positive AFB stain which was comparable to the study by $\mathrm{N}$ patel et al. Similarly, most of the granulomas were well formed and multiple which are more suggestive of tuberculosis.

The peritoneum is frequently involved in ITB. Hence performing a peritoneal biopsy or analysis of the ascitic fluid will increase the diagnostic yield. In this study either blind or USG guided ascitic fluid aspiration for biochemical analysis was done in all patients with ascites. The typical findings are increased leucocyte count with a lymphocyte predominance in low Serum Ascitic Albumin Gradient (SAAG) and high protein ascites. Most patients with tuberculous peritonitis have straw-colored ascites with leukocyte count of 150 to 4000 cells $/ \mathrm{mm}^{3}$, with a relative lymphocytic pleocytosis ${ }^{21}$. The ascitic fluid ADA has a high diagnostic value when higher cutoffs are used. ADA acts in proliferation and differentiation of lymphocyte, especially T lymphocyte and it's level increases in TB because of the stimulation of T cells by mycobacterial antigens ${ }^{22}$. When a cutoff value of $>30 \mathrm{IU} / \mathrm{L}$ is used, the sensitivity and specificity levels over $90 \%$ have been reported ${ }^{23}$. The average
ADA value of $73 \mathrm{IU} / \mathrm{L}$ with a minimum of 46 makes a strong case in favor ITB in those with concomitant peritoneal involvement.

\section{CONCLUSION}

Due to its nonspecific nature, making a diagnosis of ITB is often difficult. Delays in diagnosis is often associated with complications like perforation, abscess, fistula, strictures, and/or bleeding. Hence a composite analysis of the clinical manifestations, radiological findings, endoscopic features and finally the histopathology reports of biopsy specimens is required in making a definite diagnosis. ITB is frequently accompanied by extraintestinal involvement in the form of pulmonary lesions, intraabdominal lymphadenopathy and peritoneal involvement. Hence the evaluation of sputum samples for AFB, lymph node cytology for AFB and caseating granulomas and biochemical analysis of the ascitic fluid should be done when needed as they all increase the diagnostic yield.

\section{REFERENCES}

1. World Health Organization. Global tuberculosis report 2019. 283 p. Avialable from : https:// apps.who.int/iris/handle/10665/329368

2. Ibrarullah M, Mohan A, Sarkari A, Srinivas M, Mishra A, Sundar TS. Abdominal tuberculosis: diagnosis by laparoscopy and colonoscopy. Trop Gastroenterol. 2002; PMID: 12693163

3. Mandavdhare H., H. Singh and V. Sharma. Recent Advances In The Diagnosis And Management Of Abdominal Tuberculosis. EMJ Gastroenterol. 2017;6(1):52-60. [PDF]_emghealth.com

4. Tabrisky J, Lindstrom RR, Peters R, Lachman RS. Tuberculous Enteritis: Review of a Protean Disease. Am J Gastroenterol. 1975;63(1):49-57. PMID: 1119469

5. Anand BS, Nanda R, Sachdev GK. Response of tuberculous stricture to antituberculous treatment. Gut. 1988;29:62-9. https://doi. org/10.1136/gut.29.1.62

6. Foster BD, Buchberg B, Parekh NK, Mills S. Case of intestinal tuberculosis mimicking Crohn's disease. Am J Case Rep. 2012;13:58-61. https:// doi.org/10.12659/AJCR.882756

7. National Tuberculosis Program. Annual report. Natl Tuberc Center, Nepal. 2017;74:1-128. Available from: http://nepalntp.gov.np/wpcontent/uploads/2018/03/Final-Annual- 
Report-NTPN-2018.pdf

8. Patel N, Amarapurkar D, Agal S, Baijal R, Kulshrestha P, Pramanik S, et al. Gastrointestinal luminal tuberculosis: Establishing the diagnosis. J Gastroenterol Hepatol. 2004;19(11):1240-6. DOI: $10.1111 / \mathrm{j} .1440-1746.2004 .03485 . \mathrm{x}$

9. Gan H, Mely M, Zhao J, Zhu L. An Analysis of the Clinical, Endoscopic, and Pathologic Features of Intestinal Tuberculosis. J Clin Gastroenterol. 2016;50(6):470-5. https://doi.org/10.1097/ MCG.0000000000000514

10. Sanai FM, Bzeizi KI. Systematic review: Tuberculous peritonitis - Presenting features, diagnostic strategies and treatment. Aliment Pharmacol Ther. 2005;22(8):685-700. https:// doi.org/10.1111/j.1365-2036.2005.02645.x

11. Marshall JB. Tuberculosis of the gastrointestinal tract and peritoneum. Am J Gastroenterol. 1993 Jul;88(7):989-99. Available from: http://www. ncbi.nlm.nih.gov/pubmed/8317433. PMID: 8317433.

12. Tariq A Mir, Mir Nadeem et al. Computerised Tomography in the diagnosis of abdominal tuberculosis.Global Journal for Research Analysis. Volume -6, Issue - 12 : 2017;I(12):902. https:/ /www.doi.org/10.36106/gjra

13. Alvares JF, Devarbhavi H, Makhija P, Rao S, Kottoor R. Clinical, colonoscopic, and histological profile of colonic tuberculosis in a tertiary hospital. Endoscopy. 2005 Apr;37(4):351-6. https://doi. org/10.1055/s-2005-861116

14. Suri S, Gupta S, Suri R. Computed tomography in abdominal tuberculosis. $\mathrm{Br} \mathrm{J}$ Radiol. 1999;72(1):92-8. $\quad$ https://doi.org/10.1259/ bjr.72.853.10341698

15. Yang ZG, Min PQ, Sone S, He ZY, Liao ZY, Zhou $\mathrm{XP}$, et al. Tuberculosis versus lymphomas in the abdominal lymph nodes: evaluation with contrast-enhanced CT. Am J Roentgenol. 1999 Mar;172(3):619-23. https://doi.org/10.2214/ ajr.172.3.10063847

16. Debi U, Ravisankar V, Prasad KK, Sinha SK, Sharma AK. Abdominal tuberculosis of the gastrointestinal tract: Revisited. World J Gastroenterol. 2014;20(40):14831-40. https:// doi.org/ 10.3748/wjg.v20.i40.14831

17. Kim KM, Lee A, Choi KY, Lee KY, Kwak JJ. Intestinal tuberculosis: Clinicopathologic analysis and diagnosis by endoscopic biopsy. Am J Gastroenterol [Internet]. 1998;93(4):606-
9. $\underline{0241.1998 .173 \text { b.x }}$

https://doi.org/10.1111/j.1572-

18. Lee Y, Yang S-K, Byeon J-S, Myung S-J, Chang $\mathrm{H}-\mathrm{S}$, Hong S-S, et al. Analysis of Colonoscopic Findings in the Differential Diagnosis Between Intestinal Tuberculosis and Crohn's Disease. Endoscopy. 2006 Jun;38(6):592-7. https://doi. org/10.1055/s-2006-924996

19. Kedia S, Das P, Madhusudhan KS, Dattagupta S, Sharma R, Sahni P, et al. Differentiating Crohn's disease from intestinal tuberculosis. World J Gastroenterol. 2019 Jan;25(4):418-32. https:// doi.org/10.3748/wjg.v25.i4.418

20. Horvath KD, Whelan RL. Intestinal tuberculosis: return of an old disease. Am J Gastroenterol. 1998 May;93(5):692-6. $\quad$ https://doi.org/10.1111/ j.1572-0241.1998.207 a.X

21. Marshall JB. Tuberculosis of the gastrointestinal tract and peritoneum. Am J Gastroenterol. 1993 Jul;88(7):989-99. PMID: 8317433.

22. Barua R, Hossain M. Adenosine Deaminase in Diagnosis of Tuberculosis: A Review. Anwer Khan Mod Med Coll J. 2014;5(2):43-8. https:// doi.org/10.3329/akmmcj.v5i2.21132

23. Sanai FM, Bzeizi KI. Systematic review: Tuberculous peritonitis - Presenting features, diagnostic strategies and treatment. Aliment Pharmacol Ther. 2005;22(8):685-700. https:// doi.org/10.1111/j.1365-2036.2005.02645.x 